\title{
A Phase Transition in a System Driven by Coloured Noise
}

\author{
M. Arrayás ${ }^{(a)}$, M.I. Dykman ${ }^{(b)}$, R. Mannella ${ }^{(c)}$, \\ P.V.E. McClintock ${ }^{(a)}$, and N.D. Stein ${ }^{(a)}$ \\ ${ }^{(a)}$ Department of Physics, Lancaster University, Lancaster LA1 $4 Y B, U K$ \\ (b) Department of Physics and Astronomy, Michigan State University, East Lansing, MI 48824 \\ (c) Dipartimento di Fisica, Università di Pisa and and INFM UdR Pisa, Piazza Torricelli 2, \\ 56100 Pisa, Italy
}

\begin{abstract}
For a system driven by coloured noise, we investigate the activation energy of escape, and the dynamics during the escape. We have performed analogue experiments to measure the change in activation energy as the power spectrum of the noise varies. An adiabatic approach based on path integral theory allows us to calculate analytically the critical value at which a phase transition in the activation energy occurs.
\end{abstract}

\section{INTRODUCTION}

Gaussian white noise is a mathematical idealization which applies to a limited number of physical situations where noise has a broad power spectrum. More realistic models of random forces have also been proposed, and one of them, which is in some sense opposite to the white noise model, is quasi-monochromatic noise (QMN) $[1,2]$. In QMN, most of the power is concentrated within a relatively narrow range of frequencies.

The problem of activated escape and diffusion in far from equilibrium systems under the influence of different kinds of noise is of general interest. It has been attracting increasing attention in diverse scientific contexts, from crystal growth and current-induced desorption from crystal surfaces, to current switching in microstructures, and biological applications.

For an overdamped system in a double well potential driven by QMN, a bifurcation in the optimal path (the optimal trajectory for departure from an initial attractor) was found theoretically [3], leading to the prediction of a marked reduction in the mean escape time [1]. As we show below, related effects which involve the onset of focusing singularities and caustics, were observed in models with more than one degree of freedom acted upon by white noise [4].

CP502, Stochastic and Chaotic Dynamics in the Lakes: STOCHAOS,

edited by D. S. Broomhead, E. A. Luchinskaya, P.V. E. McClintock, and T. Mullin

(C) 2000 American Institute of Physics 1-56396-915-7/00/\$17.00 


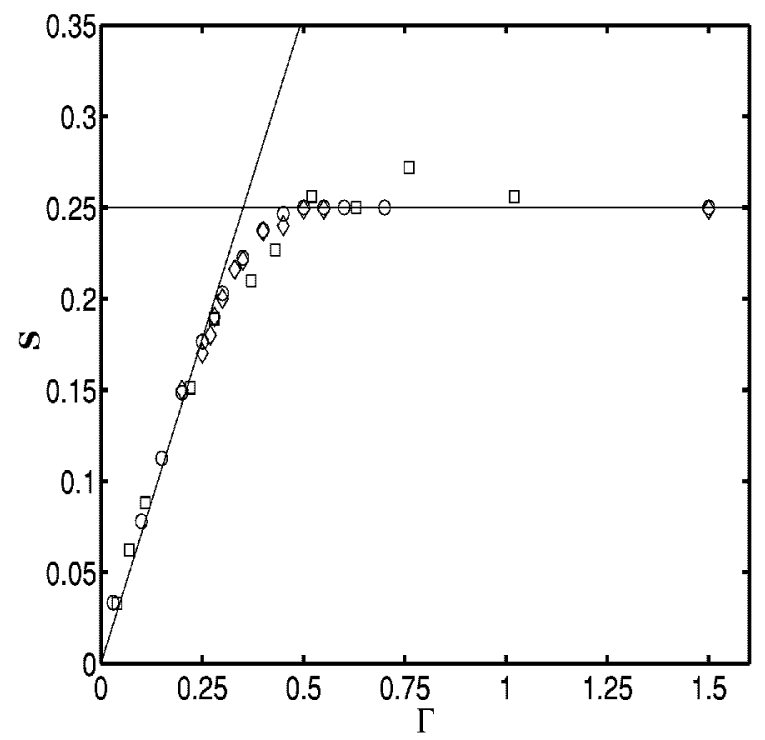

FIGURE 1. Measured and calculated values of the action $S$ as a function of $\Gamma$ for the symmetrical potential with $\omega_{0}=10$. The squares represent experimental data from analogue simulations, the diamonds are derived by solution of the nonadiabatic Hamiltonian, and the circles are obtained by solution of the adiabatic theory. The horizontal line at $S=0.25$ indicates the white noise solution, and the line through the origin represents a least-squares fit to the adiabatic theory for small $\Gamma$.

In the present paper we develop an adiabatic theory for a QMN-driven system in order to study the bifurcation in the activation energy which is, in many respects, closely analogous to a phase transition. We show that this is a "continuous" transition which corresponds to spontaneous breaking of time symmetry of the most probable paths for escape from a metastable state. We present the first experimental demonstration of this transition through use of analogue electronic simulations [5].

\section{THE MODEL}

A simple picture of QMN is the noise which results from filtering white noise through a harmonic oscillator filter with natural frequency $\omega_{0}$ and damping $\Gamma$

$$
\begin{gathered}
\ddot{f}(t)+2 \Gamma \dot{f}(t)+\omega_{0}^{2} f(t)=\xi(t) \\
\langle\xi(t)\rangle=0, \quad\langle\xi(t) \xi(s)\rangle=2 D \delta(t-s) .
\end{gathered}
$$

Here, $\xi(t)$ represents Gaussian white noise of zero mean and intensity $D$, and we assume that $\Gamma \ll \omega_{0}$. 
The stochastic dynamics of the overdamped QMN-driven system that we consider is given by

$$
\dot{x}+U^{\prime}(x)=f(t)
$$

where $U(x)$ is an arbitrary potential that we will assume to have more than one minimum. For weak noise $f(t)$, the system mostly performs small fluctuations about one of the potential minima but, occasionally, a large fluctuation occurs in which the system switches from one potential well to another. The probability $P_{\mathrm{nm}}$ of a transition $n \rightarrow m$, where $n$ and $m$ are two minima, can be obtained [1] through use of a path-integral technique $[6,7]$. The escape rate when $D$ is small can be found up to a prefactor as

$$
P\left[x_{n \rightarrow m}\right]=N \exp \left(-S\left[x_{n \rightarrow m}(t)\right] / D\right)
$$

The idea is to find the path $x(t)$ which minimises the action $S$. This path is the solution of a variational problem which relates the optimal realization of the force $\xi(t)$ (with a probability density functional which can be immediately found from (1)) and the trajectory of the coordinate (2), subject to the constraint that this trajectory starts at the potential minimum and ends at the saddle [1]. The action is the minimal value of the corresponding variational functional. We will call the minimum action the activation energy of escape, by analogy with white noise driven systems. The variational equations can be reduced to a sixth order ordinary differential equation for $x(t)$, the Euler-Lagrange equation. Einchcomb and McKane [3] showed that the problem could be recast in Hamiltonian form, which proves to be convenient both for accurate numerical calculation of the action and also for visualisation purposes. The Hamiltonian is

$$
\begin{aligned}
H(\vec{x}, \vec{p})= & \left\{\omega_{0}{ }^{4} p_{3}{ }^{2}-p_{3} \omega_{0}{ }^{2}\left(x_{2}+U^{\prime}\left(x_{1}\right)\right)+\right. \\
& \left.2 \Gamma\left(x_{3}+x_{2} U\left(x_{1}\right)^{\prime \prime}\right)+x_{3} U^{\prime \prime}\left(x_{1}\right)+x_{2}{ }^{2} U^{\prime \prime \prime}\left(x_{1}\right)\right\}+p_{1} x_{2}+p_{2} x_{3}
\end{aligned}
$$

The phase space is six dimensional, because the Euler-Lagrange equation is sixth order. The components of $\vec{x}$ turn out to be rather simple in form

$$
\vec{x} \equiv\left(x_{1}, x_{2}, x_{3}\right)=(x, \dot{x}, \ddot{x})
$$

The expressions for the generalised momenta are more complicated and we refer the interested reader to [3].

If $\Gamma$ is small, the motion of a particle driven by QMN consists of rapid oscillations superimposed on a slow motion of the centre of oscillations. This suggests [1] that we write

$$
x(t)=x_{0}+x_{+} e^{i \omega_{0} t}+x_{-} e^{-i \omega_{0} t}
$$

and replace $U(x)$ by an effective potential $V\left(x_{0}, x_{+}, x_{-}\right)$defined as 


$$
V=\frac{1}{2 \pi} \int_{0}^{2 \pi} U\left(x_{0}+x_{+} e^{i \psi}+x_{-} e^{-i \psi}\right) d \psi
$$

In this picture we obtain an effective adiabatic Hamiltonian

$$
H=\frac{1}{2} p_{0}^{2}-p_{0} V_{0}^{\prime}+\frac{1}{4} p_{+}^{2}-4 \Gamma^{2} x_{+}^{2},
$$

and the phase space is reduced from six to four dimensions. As the phase of the oscillations is arbitrary, we can set $x_{+}=x_{-}$in deriving Eq. (8).

A remarkable feature of the adiabatic Hamiltonian is its symmetry with respect to the transformation $x_{+} \rightarrow-x_{+}, p_{+} \rightarrow-p_{+}$. This symmetry follows from the form of the potential $V$, which depends only on the product $x_{+} x_{-}=x_{+}^{2}$. Therefore there always exists an extreme trajectory of (8) with $x_{+}=p_{+}=0$. However, besides this trajectory, there may exist trajectories with broken symmetry, where $x_{+} \neq$ 0 . Clearly such trajectories emerge in pairs. From (6), they correspond to fastoscillating solutions $x(t)$ of the original variational problem. When they provide the minimum to the action functional, this signals breaking of the time symmetry. Since the phase of fast oscillations is arbitrary, this bifurcation is analogous to a continuous symmetry-breaking transition.

\section{THE DOUBLE WELL POTENTIAL}

We consider the simple double-well potential

$$
U(x)=-\frac{1}{2} x^{2}+\frac{1}{4} x^{4}
$$

which has minima at $x= \pm 1$ and a saddle at $x=0$.

For this potential, we were able to confirm the numerical results reported by Einchcomb and McKane. We have performed the first experimental tests of the theory by measurement of the activation energy using an analogue circuit. The results for this symmetrical case are plotted in Figure 1.

There is a critical value of $\Gamma$ below which our adiabatic equations yield two solutions that provide extrema for the action. One of them gives a value for the action identical with that for white noise; the other corresponds to oscillatory motion. We will refer to them as the white solution and the coloured solution respectively. Above the critical value of $\Gamma$, we can see only the white solution. Einchcomb and McKane gave an estimate of $\Gamma_{c} \simeq 0.46$ for the critical value at which the bifurcation in the optimal path occurs. The analytical result which follows from (8) is $\Gamma_{c}=0.5$ [9], a value which we also found in analogue simulations [10].

Our numerical solution of Hamilton's equations utilised a shooting technique. We followed a large number of trajectories emanating from a small region of phase space surrounding the potential minimum at $x=-1$. Paths which passed through the saddle at $x=0$ were identified as optimal paths and the corresponding action 


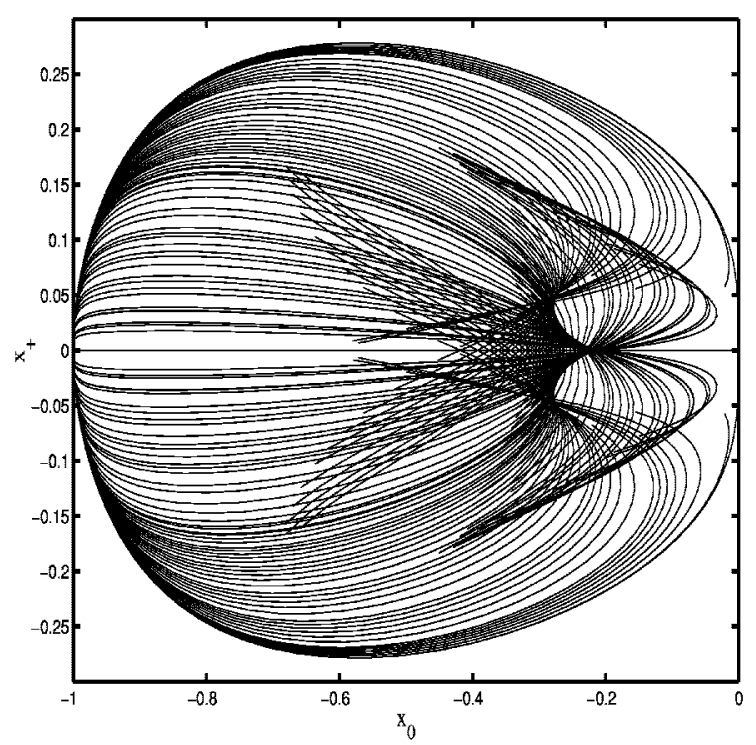

FIGURE 2. Optimal trajectories for $\Gamma=0.3$

was calculated. Three such paths can be seen in Figure 2: the x-axis and the two bounding curves. The majority of initial conditions give rise to paths which do not reach $x=0$ and therefore do not result in interwell transitions. Figure 2 shows the rather complex topology of these paths, with a cusp being clearly visible at $\left(x_{0}, x_{+}\right) \approx(-0.22,0)$.

Similar behaviour was found in the system which Maier and Stein introduced in their analysis of the escape problem in non-equilibrium systems [4]. They describe the motion of an overdamped particle in a two-dimensional field. The particle is subject to additive isotropic white noise and its position on the $(x, y)$ plane satisfies the coupled Langevin equations

$$
\begin{gathered}
\dot{x}=x-x^{3}-\alpha x y^{3}+f_{x}(t) \\
\dot{y}=-y-x^{2} y+f_{y}(t) \\
\left\langle f_{i}(t)\right\rangle=0, \quad\left\langle f_{i}(t) f_{j}(s)\right\rangle=D \delta_{i j} \delta(t-s)
\end{gathered}
$$

Since the field is not potential (unless $\alpha=1$ ) the dynamics do not satisfy detailed balance. Clearly, the system (10) has the symmetry $y \rightarrow-y$, which makes it similar to the QMN-driven system. We note that the QMN-driven system provides a natural physical realization of the system discussed by Maier and Stein. 


\section{CONCLUSIONS}

We have introduced and studied, theoretically and through analogue experiments, fluctuations in a system driven by QMN. We have been able to develop an adiabatic theory and to clarify the behaviour of the activation energy for a bistable potential as we vary $\Gamma$ keeping the frequency $\omega_{0}$ constant. The theory has made it possible to understand the nature of the transition between the known regimes of large fluctuations, which can be identified as a time symmetry-breaking transition. The symmetry-breaking in the time domain is similar to the symmetry-breaking in space observed in the system investigated by Maier and Stein.

\section{REFERENCES}

1. Dykman, M.I., Phys. Rev. A 42, 2020 (1990).

2. Dykman, M.I., McClintock, P.V.E., Stein, N.D. and Stocks N.G., Phys. Rev. Lett. 67, 933 (1991); Dykman, M.I., Mannella R., McClintock, P.V.E., Stein, N.D. and Stocks, N.G., Phys. Rev. E 47, 3996 (1993).

3. Einchcomb, S.J.B. and McKane, A.J., Phys. Rev. E 51, 2974 (1995).

4. Maier, R.S. and Stein, D.L., Phys. Rev. Lett. 71, 1783 (1993); Phys. Rev. E 48, 931 (1993); Maier, R.S. and Stein, D.L., J. Stat. Phys. 83, 291 (1996).

5. Luchinsky, D.G., McClintock, P.V.E. and Dykman, M.I., Rep. Prog. Phys. 61, 889 (1998).

6. Onsager, L., and Machlup, S., Phys. Rev. 91, 1505 (1953); Feynman, R.P., and Hibbs, A.R., Quantum Mechanics and Path Integrals, McGraw-Hill, New York, 1965; Dykman, M.I., and Krivoglaz, M.A., Soviet Phys. JETP 50, 30 (1979).

7. Bray, A.J. and McKane, A.J., Phys. Rev. Lett. 62, 493 (1989); McKane, A.J., Phys. Rev. A 40, 4050 (1989); McKane, A.J., Luckock, H.C. and Bray, A.J., Phys. Rev. A 41, 644 (1990); Bray, A.J., McKane, A.J. and Newman, T.J., Phys. Rev. A 41, 657 (1990).

8. Einchcomb, S.J.B. and McKane, A.J., Phys. Rev. E 49, 259 (1994).

9. Arrayás, M. et al, to be published.

10. Arrayás, M., Optimal Paths and Large Fluctuations, PhD thesis, Lancaster University, 1998. 
Copyright $\odot 2003$ EBSCO Publishing 
Copyright of AIP Conference Proceedings is the property of American Institute of Physics and its content may not be copied or emailed to multiple sites or posted to a listserv without the copyright holder's express written permission. However, users may print, download, or email articles for individual use. 